\title{
Estilos de apego en las personas menores víctimas de violencia de género ${ }^{6}$
}

\author{
Trabajo Resultados de Investigación \\ Olatz Soldevilla-Santamaría 7 , Alberto Blánquez-Egido ${ }^{8}$, \\ Itziar Molero-Feijoo ${ }^{9}$, Edurne Lopetegi-Alba ${ }^{10}$, \\ Cristina Pérez-Fernández ${ }^{11}$, Estibaliz Etxaniz-Ceballos ${ }^{12}$
}

\section{Introducción}

Los seres humanos, como seres sociales, forman vínculos con aquellas personas cercanas que ofrecen protección y afecto. Estos vínculos derivan en lazos afectivos más o menos estables que luego pueden extrapolarse a otro tipo de relaciones.

Ainsworth junto con Bowlby (1991), fueron los primeros investigadores interesados en analizar el establecimiento de los vínculos basándose en el estudio de la relación entre la pérdida o privación materna y el ulterior desarrollo de la personalidad, contrastándola con la teoría de la seguridad. Como consecuencia de la necesidad de buscar cercanía y contacto con las personas cuidadoras, se crean vínculos con ellas que, posteriormente, van a influir en el desarrollo de la personalidad. Gracias a los vínculos

${ }^{6}$ Filiación institucional: Asociación Educativa Berriztu, Basauri, España. E-mail: secretaria@berriztu.com

7 Experta en Traumaterapia Infanto-Juvenil Sistémica. Licenciada en Psicología.

8 Máster en Salud Mental y Terapias Psicológicas. Licenciado en Psicología.

9 Máster en Terapia Familiar Sistémica. Licenciada en Psicología.

${ }^{10}$ Máster en Psicopatología y Psicoterapia Infanto-juvenil. Licenciada en Psicología.

${ }^{11}$ Máster en Salud Mental y terapias Psicológicas. Licenciada en Psicología.

${ }^{12}$ Graduada en Psicología. 
establecidos con estas personas de referencia para el bebé se va a establecer el apego, el cual es definido por Bowlby (1990) como "una reacción de adaptación esencial para la contribución de la supervivencia de la especie”.

Además del apego, existen otras variables que van a ser mediadoras en el desarrollo de la personalidad como son: la predisposición genética, el temperamento, la familia, la educación, el proceso de socialización, el ambiente, los acontecimientos vitales y otras.

En relación al desarrollo ontogenetico del apego se ha establecido que el período más importante se sitúa entre los nueve meses y los tres años. Sobre este punto, Griffin y Bartholomew (1994) añaden que los diferentes estilos que éste puede adoptar se desarrollan de manera precoz y tienen alta probabilidad de permanecer a lo largo de toda la vida. De hecho, según Ainsworth y Bowlby (1991), esta estabilidad y continuidad del estilo de apego de cada individuo se debe a que los modelos mentales que establece sobre sí mismo y los demás que se crean gracias a ese estilo de apego y son persistentes. Estos modelos mentales al desarrollarse y actuar en un entorno familiar relativamente estable, se pueden mantener inalterables a lo largo de la vida (Musitu y Cava, 2001). No obstante, aunque tengan tendencia estable, pueden sufrir variaciones en función de los cambios acontecidos a lo largo de la vida y que modifican la conducta de cualquiera de los sujetos que conforman la relación de apego (Moreno, Del Barrio y Mestre 1996).

$\mathrm{El}$ apego adquiere mucha importancia también debido a que el vínculo que la niña o el niño forma con las personas cercanas le ayuda a aprender a regular sus emociones (Schore, 2001). El cuidador, cuidadora o figuras de referencia son los responsables de responder a las señales o reacciones emocionales de la niña o el niño y de interpretarlas y responderlas (Fonagy, Gergely y Jurist, 2004) aportando con ello seguridad al bebe. La proximidad y seguridad alcanzada gracias a la disponibilidad del cuidador principal son los principales factores que influyen en el desarrollo del apego (Hervás, 2000).

Según Lafuente (2000) "la relación afectiva paterno/materno-filial es la base de los sentimientos de seguridad o inseguridad que presiden respectivamente las vinculaciones de buena y mala calidad" (p. 166). En aque- 
llos casos en los que los lazos paterno/materno-filiales son adecuados, los individuos comienzan a sentir los primeros sentimientos positivos como seguridad, afecto y confianza (Lafuente, 2000).

En los casos en los que la proximidad y seguridad no han operado para el establecimiento de un tipo de apego seguro, se desarrollarán sentimientos negativos de inseguridad, abandono o miedo debido a que el modelo mental desarrollado suscita el temor a la inaccesibilidad de la madre. Esta es una reacción adaptativa que a lo largo de la evolución se ha transformado en primordial para la supervivencia de la especie (Bowlby, 1990).

Las investigaciones de Bowlby (1990) no solo permitieron definir el concepto de apego, sino que también sirvió como base para que posteriormente Ainsworth (1994), pudiese definir a través de sus investigaciones los tres patrones conductuales que se pueden dar en el comportamiento de los niños ante la situación de ser separados de sus madres. Gracias a lo cual, posteriormente, pudo categorizar los diferentes tipos de apego: apego seguro, inseguro-evitativo e inseguro-ambivalente.

En la situación de separación de la madre, si el menor presenta apego seguro, se comprueba la aparición de angustia de separación y reaseguramiento al volver a reunirse con la cuidadora. Se interpreta como un modelo de funcionamiento interno en el que existe confianza en el cuidador y cuya presencia reconforta (Fonagy, Gergely y Jurist, 2004). Según Botella y Corbella (2005) aquellos sujetos que han desarrollado un estilo de apego seguro son capaces de utilizar a sus cuidadores o cuidadoras como base segura ante la angustia. Esto se debe a que los sujetos son conscientes de que los cuidadores y las cuidadoras estarán disponibles y sensibles a sus necesidades. El cuidado materno en el apego seguro puede caracterizarse por estar disponible, ser receptiva, mostrar calidez y conexión.

En aquellos niños y aquellas niñas en los que se presentaba un tipo de apego inseguro-evitativo, se comprobó que no tenían confianza en la disponibilidad de la madre, mostrando poca ansiedad durante la separación y un claro desinterés en el posterior reencuentro con la madre o cuidador. Incluso en aquellos casos en los que la madre buscaba el contacto, los niños y niñas rechazaban el acercamiento (Fonagy, Gergely y Jurist, 2004). 
En el apego inseguro-ambivalente el sujeto de estudio manifiesta sentimientos de ansiedad de separación que no remiten al reunirse con la madre. A través de la observación, parece que el niño o la niña hace un intento de dramatizar el afecto para recibir la atención que ansía (Fonagy, Gergely y Jurist, 2004). El cuidado materno en estos casos se caracteriza por ser insensible, intrusivo e inconsistente. Se podría decir que el rasgo que más define a estas madres es el no estar siempre disponibles (Botella y Corbella, 2005).

Inicialmente, las investigaciones únicamente consideraban los tres tipos de apego descritos por Ainsworth (Delgado y Oliva, 2004). No obstante, más adelante otros autores han propuesto un cuarto tipo al que llaman Desorganizado-Desorientado que integra muchas de las características de los dos grupos de inseguro (Maine y Solomon, 1986). Maine y Hesse (1990) relacionaron el apego desorganizado con un ambiente asustado o de cierto miedo. Este patrón de apego aparece con frecuencia en niños cuyos cuidadores inspiran miedo, por lo que se encuentran frente a la paradoja de necesitar protección de las figuras que a su vez les provocan circunstancias atemorizantes (Maine y Hesse, 1990). En una investigación posterior Lyons-Ruth y Jacobvitz (1999) comprobaron que la desorganización en el apego puede ser predicha por una figura atemorizante del cuidador o cuidadora. El apego desorganizado parece ser un factor general de riesgo que favorece el desarrollo de la conducta desadaptada (Riggs, Jacobovitz y Hazen, 1999).

Existen evidencias que revelan que los diferentes tipos de apego están relacionados con ciertas patologías o problemas psicológicos (Girón, Rodríguez y Sánchez, 2003; Rodríguez-Testal, Carrasco, Del Barrio, y Catalán, 2002; Simpson, Rholes y Phillips, 1996). Eso es debido a que las relaciones con figuras de apego posibilitan una construcción del mundo y de sí mismo, y, por tanto, dependiendo del tipo de apego que se tenga, se actúa, se comprende la realidad o se anticipa al futuro de diferentes maneras (Bradley y Cafferty, 2001). Además, siguiendo a Bradley y Cafferty (2001) estos modelos internos regulan nuestros procesos cognitivos y emocionales mediante la selectividad de la atención, la creación de sesgos en la codificación y recuperación de los recuerdos y la influencia sobre los proce- 
sos de atribución de significado. A continuación se presentan los resultados de algunos estudios que han relacionado el tipo de apego con patologías o problemas psicológicos.

\section{Apego seguro}

Aquellas personas con tipo de apego seguro están más capacitadas para establecer relaciones tienen una mayor capacidad para intimidar y mostrar afecto con sus iguales. Esto se debe a que han sido capaces de desarrollar modelos mentales de sí mismos con características positivas como amigables, afables y capaces, mientras que a los otros los perciben como confiables y bien intencionados (Botella y Corbella, 2005).

Este estilo de apego supone un factor de resiliencia psicológica ya que impulsa la competencia social, el funcionamiento cognitivo, el bienestar emocional y la capacidad de superación frente a la adversidad (Siegel, 1999). Además, según Lafuente (2000) aquellos adultos con un estilo de apego seguro tienen más alta autoestima, son socialmente más activos y presentan menos soledad que los individuos con un apego inseguro ambivalente.

El apego seguro también tiene consecuencias positivas en el desarrollo cognitivo, entre otras cosas: mejor desarrollo intelectual (Jacobsen y Hoffmann, 1997); mejores capacidades en algunos aspectos del lenguaje (Vanljzendorn, 1995); tienen más habilidad para el juego simbólico (Meins, 1997); son más flexibles, abiertos, positivos y realistas en el procesamiento de la información, Kirsch y Cassidy (1997); poseen mayor inteligencia social (Frankel y Bates, 1990); y en la etapa adulta su rendimiento laboral es mejor (Hardy y Barkham , 1994).

\section{Apego inseguro y desorganizado}

El desarrollo de desórdenes emocionales y conductuales en la adolescencia parece estar relacionado con los cambios familiares que amenazan la disponibilidad de las figuras de apego, así como con la aparición de trastornos de personalidad en los adultos jóvenes (Stein, Marton, Golombeck y Korenblum, 1994). Las personas con estilo de apego inseguro tienden a desarrollar modelos de sí mismos y del resto que a su vez son también inseguros caracterizando a los demás y las relaciones como poco confiables. 
Esto es así debido en parte a que este tipo de personas refleja una frecuente preocupación por la posibilidad del abandono (Bowlby, 1990).

Con respecto al estilo de apego inseguro podemos decir que supone un factor de riesgo a la hora de manifestar problemas psicológicos (Siegel, 1999) ya que la psicopatología puede estar relacionada con la incapacidad de las personas de aceptar, integrar y tratar adecuadamente sus sentimientos y necesidades primarias a lo largo del desarrollo (Botella, 1994). Por otra parte, las personas pueden desarrollar unas visiones y creencias negativas sobre sí mismos o mismas y sobre el mundo también debido a la frustración repetida ante la necesidad que no es cubierta (Rodríguez-Testal, Carrasco, Del Barrio, y Catalán, 2002). Se ha constatado una relación entre el apego inseguro y la depresión en la infancia, así como con sintomatología asociada a las dificultades para las relaciones interpersonales y el comportamiento hostil en la adolescencia (Weinfield, Sroufe, Egeland y Carlson, 1999).

Aquellas personas con un estilo de apego inseguro evitativo desarrollan modelos de sí mismos basados en la suspicacia, el escepticismo y el retraimiento, y modelos de los otros como poco confiables o demasiado ansiosos para comprometerse. Estos modelos dificultan la confianza y la capacidad de depender de los otros (Simpson, Rholes y Phillips, 1996). Asimismo, se ha comprobado que los adolescentes con una organización de apego inseguro evitativo tenían más posibilidades de desarrollar problemas de conducta, abusos de sustancias trastorno de personalidad narcisista o antisocial, y rasgos paranoicos de personalidad (Girón, Rodríguez y Sánchez, 2003).

Por otra parte, los tipos de apego inseguro se han relacionado con el trastorno límite de la personalidad debido a la presencia de traumas no resueltos en las personas que lo padecen (Aaronson, Bender, Skodol y Gunderson, 2006), así como también se ha relacionado la aparición de este trastorno con la existencia del estilo de apego desorganizado (Westen, Nakash, Thomas y Bradley, 2006). Los estudios de Agrawal, Gunderson, Holmes, y Lyons-Ruth (2004) demuestran que entre un 50 y un $80 \%$ de los pacientes con trastorno límite de la personalidad reúnen características de al menos uno de estos estilos de apego. 
Existe una relación entre el apego inseguro ambivalente en la infancia y la psicopatología ya que este tipo de apego se relaciona con el trastorno de ansiedad en el adolescente (Warren, Huston, Egeland y Sroufe, 1997).

Por su parte, el estilo de apego desorganizado mantiene una relación con la aparición de agresividad infantil o reacciones violentas (Shaw, Owens, Vondra, Keenan y Winslow, 1996).

Se han revisado estudios a cerca de menores que han sufrido malos tratos que señalan que entre un 15 y 23\% de ellos desarrollan un estilo de apego inseguro evitativo. Alrededor de un $20 \%$ de esta población presenta un estilo de apego inseguro ambivalente y aparecen rasgos compatibles con el apego desorganizado en un 75-80\% de estos menores (Barudy y Dantagnan, 2005).

Teniendo en cuenta que estos menores maltratados son víctimas de violencia de manera directa se considera que, ya que en el marco legal del estado español los menores expuestos a violencia de género son también considerados víctimas de la violencia, ambos grupos de menores podrían compartir características psíquicas, dada su vivencia de la violencia. Por ello, se hipotetiza sobre el hecho de que los menores víctimas de la exposición a la violencia de género pudieran estar también desarrollado estilos de apego no-seguros.

Dicho lo cual, el objetivo de este estudio descriptivo es observar en qué medida los tipos de apego inseguro ambivalente, inseguro evitativo y desorganizado aparecen en la muestra de niñas y niños expuestos a violencia de género atendidos desde la Asociación Educativa Berriztu. Cumplir con este objetivo debe orientar a los profesionales de la atención psicoterapéutica a menores víctimas de violencia de género sobre el modo en como la vivencia de este tipo de violencia puede posteriormente favorecer la aparición de sintomatología compatible con patología mental. De este modo se considera que se puede colaborar con el establecimiento y desarrollo de estrategias terapéuticas que favorezcan una relación de ayuda de calidad entre el sujeto ayudado y el ayudador. 


\section{Metodología}

El presente estudio tiene como planteamiento principal la descripción de las variables propias de la muestra seleccionada.

Por un lado la descripción cualitativa del tipo de apego de cada uno de los sujetos de la muestra a través de la utilización de la adaptación de la prueba Proyectiva Pata Negra (Corman, 1981): PNO-Ap (Loizaga et al, 2016). El cómputo cuantitativo de la aparición de los diferentes tipos de apego tiene el objeto de plasmar las tendencias existentes en esta muestra de la población de estudio que en futuro podrían ser inferibles a la totalidad de la misma en estudios que sigan una posible línea de investigación.

Por otra parte, la aportación de variables cuantitativas discretas de los valores en las diferentes dimensiones del test SENA (Fernández-Pinto, et al., 2015) pretende descubrir en qué medida aparecen rasgos psicopatológicos en los individuos que forman de la muestra y orientar sobre la posibilidad de que repeticiones recurrentes en dichos rasgos pudieran arrojar luz sobre la posibilidad de que determinadas patologías tengan lugar con mayor probabilidad en niñas y niños expuestos a Violencia de Género.

El nivel descriptivo y de diseño sencillo de este estudio pretende tener un alcance reducido tanto en cuanto pretende únicamente descubrir posibles tendencias dentro de la muestra obtenida que describan el tipo de apego predominante dentro de la misma y las tendencias psicopatológicas existentes dentro del grupo de menores expuestos a Violencia de Género que han sido sujetos de esta iniciativa.

\section{Muestra}

Los sujetos de estudio son en todo caso personas ayudadas por la Asociación Educativa Berriztu a través del Centro Txikiak (Espacio de Bienestar Psicosocial para menores Víctimas de Violencia de Género) conveniado con el Departamento de Mujer y Familia de la Sección de Acción Social de la Diputación Foral de Bizkaia.

La muestra de 24 sujetos $(\mathrm{N}=24)$ es de tipo intencional habiendo sido obtenida de manera no probabilística y estando formada por aquellos menores que han acudido al Centro Txikiak en su primer año de funciona- 
miento y que han sido incluidos en el estudio con el consentimiento de sus progenitores.

La muestra está formada por sujetos que han sido víctimas de Violencia de Género y estaban siendo ayudadas y ayudados en el Centro Txikiak (Asociación Educativa Berriztu) en el momento de la pasación de las pruebas. En el momento de su participación en el estudio ninguno de los menores estaba expuesto a Violencia de Género.

Los sujetos pertenecen a ambos sexos a razón de 5 niñas $(n=5)$ y 19 niños ( $n=19)$, de entre 5 y 18 años, que han sido distribuidos en los 3 rangos de edad propuestos en el test SENA de tal manera que los grupos se distribuyen en los espacios comprendidos desde los 3 hasta los 6 años, con 4 sujetos $(n=4)$, desde los 6 hasta los 12 años con 12 sujetos $(n=12)$ y a partir de 12 años hasta los 18,6 sujetos $(n=8)$.

\section{Procedimiento}

Todos los sujetos que forman parte de la prueba han sido admitidos en el Centro Txikiak al considerarse previa evaluación de las personas técnicas especialistas oportunas de la Asociación Educativa Berriztu y la Diputación Foral de Bizkaia que presentan sintomatología compatible con haber estado expuestos a situaciones de Violencia en el hogar. Todos ellos han sido incluidos en el programa habiéndose firmado los consentimientos oportunos por parte de los progenitores custodios de estos menores y no existiendo negativa a tal inclusión por parte de los progenitores no custodios acorde a lo establecido por las regulaciones legales de la actividad.

Todos los sujetos acceden a un proceso en el que reciben ayuda psicoterapéutica individual directa y los adultos relacionados estrechamente con estos sujetos reciben una asistencia socioeducativa abordándose las situaciones particulares de cada menor con una perspectiva familiar.

El proceso se compone de una primera parte denominada Fase de $\mathrm{Ob}$ servación que dura 4 sesiones y que da paso posteriormente a una Fase de Intervención en la que la psicoterapia sigue su curso en base a lo observado en ese primer momento. Durante la Fase de Observación el psicoterapeuta lleva a cabo una Entrevista de Desarrollo Evolutivo con la madre del sujeto ayudado. 
En el momento del cambio de Fase se informó a los adultos de referencia del menor de su inclusión en el estudio y se les pasó un consentimiento informado. A las hijas e hijos de aquellas que aceptaron, se les administró en primera instancia el SENA (Fernández-Pinto, et al., 2015) y posteriormente el PNO-Ap (Loizaga et al, 2016) en sesiones diferenciadas.

Ambas posiciones tienen lugar en la $5^{\mathrm{a}}$ y la $6^{\mathrm{a}}$ sesión del proceso psicoterapéutico en curso en todos los casos.

Posteriormente, se procede a la corrección de las pruebas de forma que el test SENA se corrige gracias a la aplicación informática desarrollada a tal efecto por TEA-Ediciones. La corrección del PNO-Ap se realiza por parte del psicoterapeuta responsable del proceso psicoterapéutico del menor que también ha sido el responsable de aplicarle la prueba al menor y posteriormente se realiza una corrección de lo recogido en la pasacion por parte del equipo de psicoterapeutas que forma parte del Centro Txikiak en su totalidad.

Tras lo cual se sistematizan los datos y se analizan dando pie a las conclusiones que posteriormente se expondrán.

\section{Materiales}

Para llevar a cabo el estudio se utilizaron diferentes materiales. Test de Pata Negra Objetivado-Apego (Loizaga et al, 2016) y el Sistema de Evaluación de Niños y Adolescentes SENA (Fernández-Pinto, et al., 2015).

El primero es una adaptación del test proyectivo Pata Negra de Corman (1981). Las láminas del test PN evocan temas que hacen referencia a los conflictos emocionales propios del desarrollo, relacionados especialmente con la percepción de las relaciones entre el niño y su padre, madre y hermanos y hermanas.

Aunque el test consta de 17 láminas, en esta adaptación solo se utilizaron 8, que fueron las siguientes: para medir las primeras relaciones se utilizó la lámina I, para medir las relaciones con las figuras de referencia se utilizaron la lámina II y V, para medir la pérdida y separación de las figuras de apego versus independencia se utilizaron la lámina III y IV, para 
las relaciones castigo/culpabilidad y agresividad hacia las figuras parentales se utilizaron la lámina VI y VIII, y por último, para medir otras figuras de apego se utilizó la lámina VIII. En la adaptación de Loizaga y colaboradores (2016), se define para cada lámina qué situaciones evocadas por la niña o el niño se identificarían como apego seguro, inseguro-evitativo o inseguro-ambivalente.

La administración de las láminas se presenta cada una de las 8 en orden $\mathrm{y}$ se les pide que realicen una historia narrada en presente, pasado y futuro en cada una de ellas, además de pedirle que se identifique con alguno de los personajes o con nadie.

Como prueba de la validez y fiabilidad de esta prueba para el presente estudio se ha llegado a un acuerdo entre los jueces en todos los casos en virtud del tipo de pasación y corrección por el que se ha optado y siguiendo los criterios propuestos por los autores del PNO-Ap.

E1 SENA es una prueba psicométrica que propone un sistema de evaluación aplicable a niñas y niños de entre los 3 y los 18 años. Este sistema es multifuente contando con el Autoinforme que rellena el sujeto y el informe de la Familia y de la Escuela lo rellenan personas pertenecientes a ambos contextos relevantes para el menor. En este caso se ha utilizado la prueba de autoinforme para los menores.

La prueba establece 3 niveles para adaptarse a las características y problemas más relevantes en cada edad: Nivel 1 (Infantil, 3 a 6 años), Nivel 2 (Primaria; 6 a 12 años) y nivel 3 (Secundaria; 12 a 18 años).

La administración del Autoinforme tiene lugar en la sala de terapia en presencia del psicoterapeuta del menor el cual es el encargado de facilitar la prueba al menor y darle las instrucciones para su realización. El administrador permanece presente durante toda la prueba con el objeto de poder resolver posibles dudas que surjan en la comprensión de los enunciados.

El tiempo de aplicación estimado del autoinforme es de 20 a 30 minutos y su finalidad es realizar una evaluación de un amplio espectro de problemas emocionales y de conducta, problemas contextuales, así como áreas de vulnerabilidad y recursos psicológicos. 
La validez de las diferentes dimensiones que forman parte del Autoinforme del test SENA para los 3 grupos de edad obtiene en todos ellas un alfa de Cronbach superior al .70 ( $\alpha \geq .70)$ y una fiabilidad test-retest de entre .58 y .93 .

\section{Análisis de datos}

\section{Herramientas de recogida y análisis de datos.}

Para la recogida y organización de los datos obtenidos mediante la utilización de ambas pruebas se utilizaron Tablas estandarizadas para la evaluación del PNO-Ap (Loizaga et al, 2016) y el informe resultante de la corrección informatizada del SENA (Fernández-Pinto, et al., 2015).

A partir de esta ordenación de los datos, estos han sido transferidos a hojas de cálculo creadas ad hoc para la posterior operacionalización de los mismos.

En relación al PNO-Ap, gracias a la interpretación de lo expresado por los menores en el momento de realizar la prueba en cada una de las láminas de que se compone se ha otorgado un valor numérico a cada uno de los diferentes tipos de apego en cada una de esas láminas. Posteriormente el sumatorio de las puntuaciones otorgadas por los evaluadores permite cotejar los resultados totales para cada tipo de apego y definir cuál es el predominante siguiendo el modelo de corrección propuesto por el autor (Loizaga y colaboradores, 2016). Este apego predominante es el que se ha definido como el principal para cada sujeto y el único que se ha tenido en cuenta en adelante.

Los diferentes tipos de apego han sido codificados numéricamente para su operacionalizacion a través de las hojas de cálculo.

De la misma forma se introdujeron en las hojas de cálculo para su operacionalizacion las puntuaciones tipificadas obtenidas a través del informe generado por la aplicación informática del SENA. Estas puntuaciones tipificadas son las resultantes a partir de la introducción manual de los datos obtenidos a través de la administración en papel de la prueba Autoinforme en el sistema informático encargado de corregir el test. 
Los datos obtenidos a través de las Tablas, se codificaron en una matriz en Excel y después se exportaron a SPSS para facilitar su análisis.

\section{Procedimientos de análisis de datos.}

El análisis de los datos se realizó con el programa informático estadístico SPSS en su versión 22. Las matrices de datos realizadas ad hoc fueron utilizadas para obtener estadísticos descriptivos sobre las variables de estudio y observar las posibles relaciones entre el tipo de apego y las dimensiones del SENA (Fernández-Pinto, et al., 2015).

\section{Resultados}

En lo que se refiere a la presencia de los diferentes tipos de apego se obtuvo, como se puede observar en el gráfico 1, que el tipo de apego prevalente ha sido el inseguro evitativo ( $50 \%$ de los sujetos), $(n=12)$, seguido del inseguro-ambivalente (25\%), $(\mathrm{n}=6)$; seguro $(17 \%),(\mathrm{n}=4)$ y desorganizado $(8 \%),(n=2)$.

Gráfico 1. Porcentajes de los tipos de apego

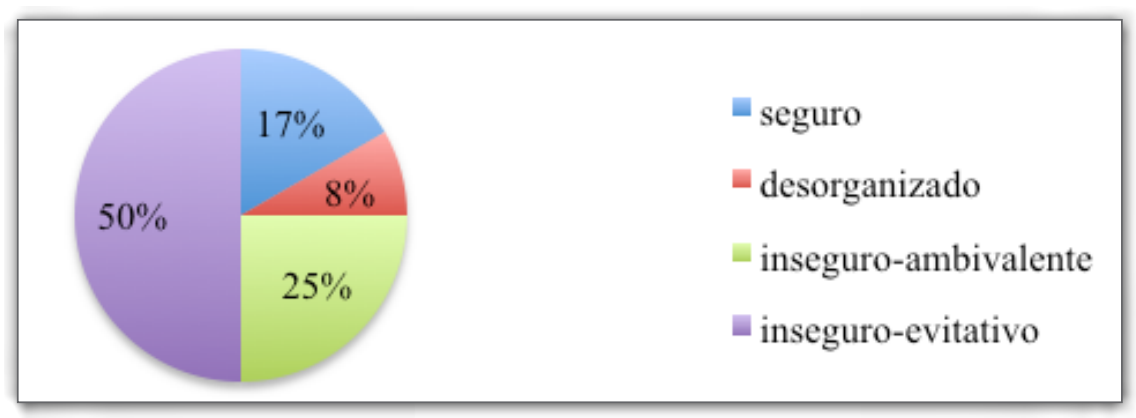

Fuente: Elaboración propia.

Otra de las cuestiones que se observó fueron las diferencias entre los géneros. Se puede observar, que tanto las niñas como los niños en su mayoría se sitúan en el eje inseguro ambivalente o inseguro evitativo. A diferencia de los chicos, ninguna de las chicas presentó un tipo de apego seguro (Véase gráfico 2). Se obtiene que los chicos se distribuyen de manera que 4 de ellos tienen apego seguro, 2 apego desorganizado, otros 4 cuentan con apego inseguro-ambivalente y por último 9 sujetos varones tienen un apego 
de tipo inseguro evitativo. En lo referido a las chicas, 2 de ellas tendrían un apego de tipo inseguro-ambivalente y las otras 3 poseerían un apego inseguro evitativo.

Gráfico 2. Diferencias en los tipos de apego en función del género

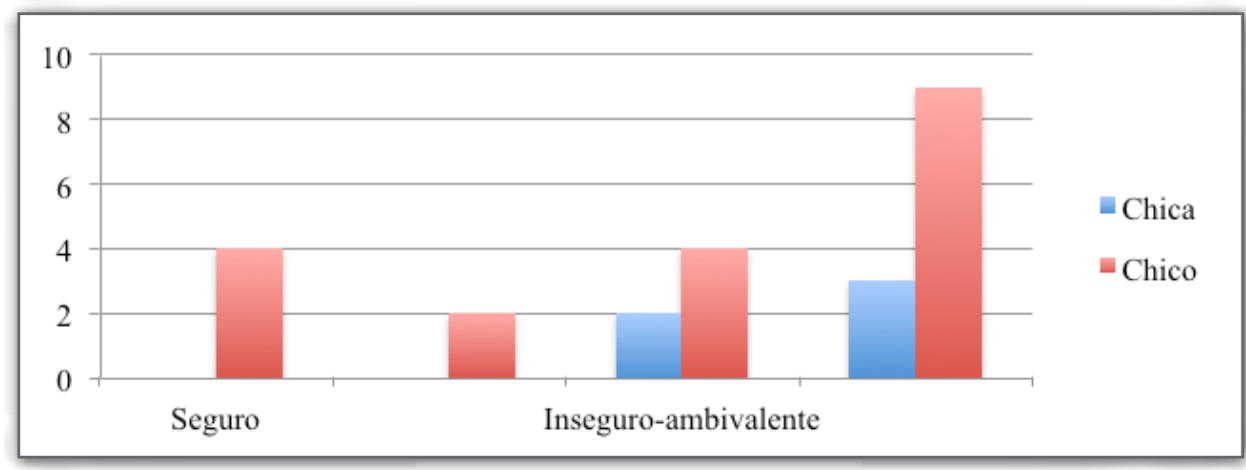

Fuente: Elaboración propia.

En el gráfico 3 se puede observar las diferencias en los tipos de apego en función de los tres grupos de edad establecidos: de 3 a 6 años, de 7 a 12 años y de 13 a 18 años.

Gráfico 3. Diferencias en los tipos de apego en función de los grupos de edad

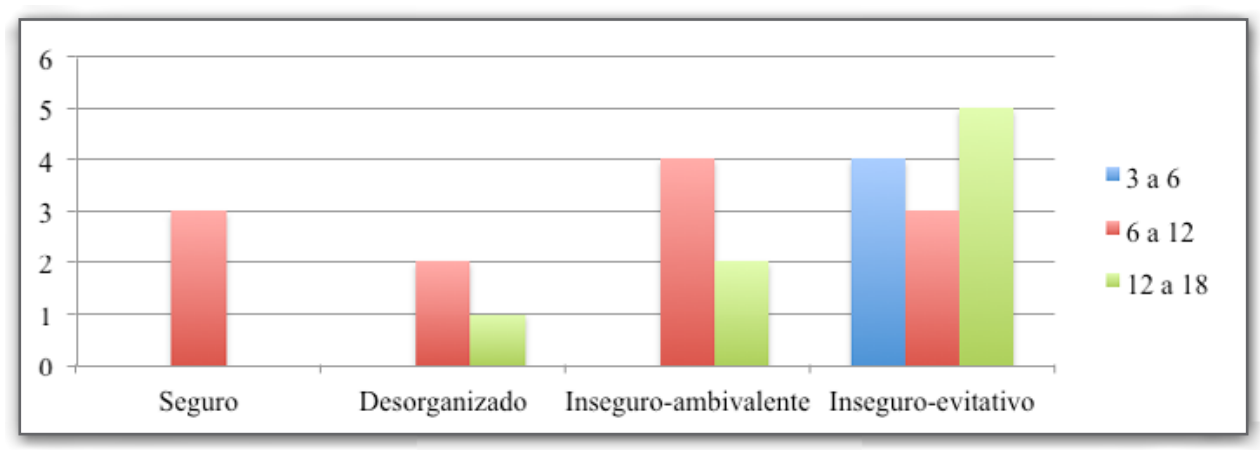

Fuente: Elaboración propia.

Como se puede observar en el gráfico 3, el número total de sujetos con Apego Seguro pertenecen al rango de 6 a 12 años, todas aquellas niñas y 
aquellos niños que se sitúan en el rango de 3 a 6 años tienen un tipo de apego inseguro-evitativo. Mientras que los sujetos que se encuentran entre 6 y 12 años se reparten de manera similar entre los 4 tipos de apego. Por último, los individuos que pertenecen a las edades comprendidas entre los 12 y los 18 se reparten entre los tipos desorganizado, inseguro-ambivalente e inseguro-evitativo.

En cuanto a la relación entre el apego predominante en los sujetos y las variables del SENA (Fernández-Pinto, et al., 2015), se han observado tendencias de relación entre los diferentes tipos de apego y algunas variables. A la luz de los resultados se puede indicar que se ha observado que aquellos sujetos que tienen el tipo de apego inseguro ambivalente tienen las puntuaciones más altas en depresión, problemas emocionales, ansiedad, recursos personales y problemas de regulación emocional. Aquellas variables en las que los sujetos con apego desorganizado puntuaron más alto fueron; problemas conductuales, de atención y de control de la ira.

\section{Discusión}

En los sujetos que han sufrido exposición a la violencia de género que están incluidos en el programa, quiere decir, que ya se ha observado que presentan sintomatología compatible con la misma, se observa que han presentado preferentemente un tipo de apego inseguro-evitativo. Esto nos puede llevar a pensar que personas con ese tipo de apego pueden ser más vulnerables a la vivencia de situaciones de violencia de género. Si bien, no se han encontrado referencias al respecto en la literatura revisada, pero se considera que es una idea que podría servir para enriquecer el planteamiento de futuras investigaciones.

Sobre la relación entre el tipo de apego de los sujetos y las posibles patologías a desarrollar a lo largo de su vida, podemos concluir a la luz de los resultados obtenidos que se observa una tendencia en aquellos sujetos de la muestra a los que se les atribuyó un tipo de apego inseguro ambivalente o desorganizado a mostrar puntuaciones más altas en varias de las escalas del SENA (Fernández-Pinto, et al., 2015) (Ver Anexo 1). Esto concuerda con la literatura previa. 
Tal es el caso de la relación entre el apego inseguro y la depresión, ya que según Rodríguez-Testal et al. (2002) "aquellas personas con un tipo de apego inseguro tienden a tener visiones y creencias negativas sobre el mundo y sobre uno mismo también". También Weinfield et al. (1999) sugerían que "el apego inseguro se relaciona con la depresión en la infancia". Habiendo encontrado que los sujetos con Apego Inseguro puntúan de manera significativa en la dimensión de depresión del SENA, el actual estudio refuerza las ideas expuestas en la literatura previa de referencia.

Con respecto al tipo de apego desorganizado también se observan similitudes con estudios previos que sugerían que aquellas personas con un tipo de apego desorganizado eran más susceptibles a desarrollar problemas de conducta (Girón et al., 2003), tal y como se puede observar relacionando los resultados obtenidos entre ambas variables en el presente estudio.

De manera más amplia y teniendo en cuenta la necesidad de acudir a un nivel de correlación entre el tipo de apego de los sujetos y las dimensiones del SENA para obtener resultados significativamente relevantes, es preciso reseñar que las observaciones realizadas sobre los resultados del análisis de datos se han realizado de una manera dialogada entre los jueces responsables de la pasacion y corrección del PNO-Ap (Loizaga et al, 2016) y SENA. Se ha llegado a acuerdos que señalan que es factible que la relación entre el tipo de apego y la puntuación significativa de los sujetos en determinadas dimensiones del SENA puede tener base para poder realizar estudios futuros que traten de corroborar esas relaciones a través de la correlación de las variables obtenidas a través de los resultados obtenidos tras la pasacion y corrección de dichas pruebas en el futuro. La comprobación o no de las hipótesis en esta línea que se generan en el equipo responsable del estudio se considera que pueden ser de relevancia para describir una parte de la realidad psíquica de la población de niños y niñas víctimas de violencia de género que pueda orientar nuevas líneas de investigación o tratamiento que les ayuden a paliar las consecuencias sintomáticas asociadas a la exposición a la violencia de género que hayan podido sufrir.

Asimismo, el estudio actual tiene más limitaciones a tener en cuenta en el futuro a la hora de seguir esta línea de investigación que actualmente se encuentra en su etapa primigenia en las entidades precursoras de este 
estudio. En primer lugar, la validez y fiabilidad del instrumento utilizado para categorizar el apego ( $\mathrm{PNO}-\mathrm{Ap}$ ) no han sido estudiadas aún en profundidad. Además, solo se ha considerado el tipo de apego prevalente y no qué porcentaje de cada tipo de apego tenía cada sujeto. Por otro lado, las medidas se realizaron en un único momento temporal y al comienzo de la interacción psicoterapéutica, lo que puede implicar cierta distorsión de los resultados.

El presente estudio ofrece información descriptiva sobre una muestra perteneciente a una población muy concreta y nos acerca a la realidad de las niñas y niños víctimas de violencia de género. Pero es preciso reseñar que el hecho de que la muestra se haya obtenido de una manera no probabilística impide que se pueda tomar esta muestra como representativa de la población total de niños y niñas víctimas de violencia de género generalizándose los resultados obtenidos y las hipotéticas tendencias de relación entre variables. Por lo tanto, este estudio permite iniciar una previsión sobre el funcionamiento psíquico que pueden tener en un futuro cercano o más lejano las niñas y niños pertenecientes a la muestra y orientar a las y los profesionales sobre los perfiles de los sujetos ayudados que acuden al servicio favoreciendo una mayor calidad en la ayuda psicoterapéutica.

Se puede concluir que a través de este estudio se ha podido comenzar a conocer mejor los efectos sobre los sujetos de la exposición a la violencia de género en función del tipo de apego de cada uno de ellos.

De cara a futuras investigaciones sería conveniente analizar si los resultados son estadísticamente significativos ya que en este estudio solo se ha hecho un análisis meramente descriptivo de los resultados en una muestra muy reducida de sujetos.

En cuanto a la atención psicoterapéutica de los menores incluidos dentro del programa Txikiak de la Asociación Educativa Berriztu y auspiciado por la Diputación Foral de Bizkaia, la observación de estos datos ha sido relevante de cara a orientar la mirada sobre los menores como sujetos de atención especializada teniendo en cuenta su particular realidad y generando en el equipo una mayor sensación de particularidad de la problemática que sufren siendo está altamente sensible. 


\section{Referencias bibliográficas}

Aaronson, C. J., Bender, D. S., Skodol, A. E., y Gunderson, J. G. (2006). Comparison of attachment styles in borderline personality disorder and obsessive-compulsive personality disorder. Psychiatric Quarterly, 77(1), 69-80.

Agrawal, H. R., Gunderson, J., Holmes, B. M., y Lyons-Ruth, K. (2004). Attachment studies with borderline patients: A review. Harvard review of psychiatry, 12(2), 94-104.

Ainsworth, M. S., y Bowlby, J. (1991). An ethological approach to personality development. American Psychologist, 46(4), 333-341.

Barudy, J., y Dantagnan, M. (2005). Los buenos tratos a la infancia: parentalidad, apego y resiliencia. Editorial Gedisa.

Botella, L., y Corbella, S. (2005). Neurobiología de la autorregulación afectiva, patrones de apego y compatibilidad en la relación terapeutapaciente. Revista de psicoterapia, 61(16), 77-104.

Bowlby, J. (1990). Charles Darwin: a biography. London: Hutchinson xiv, 511p.-maps, ports. En Geog, 1, 1809-1882.

Bradley, M.J., y Cafferty, T. P. (2001). Attachment among older adults: Current issues and directions for future research. Attachment y buman development, 3(2), 200-221.

Corman. L. (1981). El test P.N. Manual I. Barcelona: Herder.

Delgado, A. O., y Oliva, A. (2004). Estado actual de la teoría del apego. Revista de Psiquiatría y Psicología del Niño y del Adolescente, 4(1), 65-81.

Fernández-Pinto, I. Santamaría, P., Sánchez-Sánchez, F., Carrasco, M., y Del Barrio, V. (2015). SENA: Sistema de Evaluación de Niños y Adolescentes.

Fonagy, P., Gergely, G., y Jurist, E. L. (Eds.). (2004). Affect regulation, mentalization and the development of the self. Karnac books. 
Frankel, K. A., y Bates, J. E. (1990). Mother-Toddler Problem Solving: Antecedents in Attachment, Home Behavior, and Temperament. Child development, 61(3), 810-819.

Girón, S., Rodríguez, R., y Sánchez, D. (2003). Trastornos de comportamiento de los adolescentes. Observaciones desde una perspectiva sistémica-relacional. Psiquis, 24(1), 1-10.

Griffin, D. W., y BArtholomew, K. (1994). Models of the self and other: Fundamental dimensions underlying measures of adult attachment. Journal of personality and social psychology, 67(3), 430.

Hardy, G. E., y Barkham, M. (1994). The relationship between interpersonal attachment styles and work difficulties. Human Relations, 47(3), 263-281.

Hervás, N. (2000). El sistema de apego en la generación de los padres y terapia familiar. Systémica, (8), 89-100.

Jacobsen, T., y Hofmann, V. (1997). Children's attachment representations: longitudinal relations to school behavior and academic competency in middle childhood and adolescence. Developmental psychology, 33(4), 703.

Kirsh, S. J., y Cassidy, J. (1997). Preschoolers' Attention to and Memory for Attachment-Relevant Information. Child development, 68(6), 11431153.

Lafuente, M. J. (2000). Patrones de apego, pautas de interacción familiar y funcionamiento cognitivo (de los 70 a los 90). Revista de Psicología General y Aplicada, 53(1), 165-190.

Loizaga, F. y colaboradores (2016). Evaluación del apego-attachment y los vinculos familiares. Editorial CCS.

Lyons-Ruth, K., y Jacobvitz, D. (1999). Attachment disorganization: Unresolved loss, relational violence, and lapses in behavioral and attentional strategies. In J. Cassidy y P. R. Shaver (Eds.), Handbook of attachment: Theory, research, and clinical applications (pp. 520-554). New York: Guilford Press. 
Main, M., y Hesse, E. (1990). Parents' unresolved traumatic experiences are related to infant disorganized attachment status: Is frightened and/or frightening parental behavior the linking mechanism? In M. T. Greenberg, D. Cicchetti, y E. M. Cummings (Eds.), The John D. and Catherine T. MacArthur Foundation series on mental health and development. Attachment in the preschool years: Theory, research, and intervention (pp. 161-182). Chicago: University of Chicago Press.

Main, M., y Solomon, J. (1986). Discovery of an insecure-disorganized/ disoriented attachment pattern. In T. B. Brazelton y M. W. Yogman (Eds.), Affective development in infancy (pp. 95-124). Westport, CT: Ablex Publishing.

Meins, E. (1997). Security of attachment and maternal tutoring strategies: Interaction within the zone of proximal development. British Journal of Developmental Psychology, 15(2), 129-144.

Moreno, C., Del Barrio, V., y Mestre, M.V. (1996). Acontecimientos vitales y depresión en adolescentes. Iberpsicología: Revista Electrónica de la Federación española de Asociaciones de Psicología, 1(1), 10.

Musitu, G., y Cava, M. J. (2001). Autoestima y percepción del clima escolar en niños con problemas de integración social en el aula. Revista de Psicología general y aplicada, 54(2), 297-311.

Riggs, S. A., Jacobovitz, D., y Hazen, N. (2002). Adult attachment and history of psychotherapy in a normative sample. Psychotherapy: Theory, Research, Practice, Training, 39(4), 344-353.

Rodríguez-Testal, J. F., Carrasco-Ortiz, M. Á., Barrio-Gándara, V. D., y Catalán, M. C. (2002). Errores cognitivos en jóvenes y su relación con la sintomatología depresiva. Anuario de psicología, 33(3), 409-432.

Schore, A. N. (2001). Effects of a secure attachment relationship on right brain development, affect regulation, and infant mental health. Infant mental health journal, 22(1-2), 7-66.

Shaw, D. S., Owens, E. B., Vondra, J. I., Keenan, K., y Winslow, E. B. (1996). Early risk factors and pathways in the development of early 
disruptive behavior problems. Development and Psychopathology, 8(4), 679-699.

Siegel, D. J. (1999). The developing mind (Vol. 296). New York: Guilford Press.

Simpson, J. A., Rholes, W. S., y Phillips, D. (1996). Conflict in close relationships: an attachment perspective. Journal of personality and social psychology, 71(5), 899.

Stein, B. A., Marton, P., Golombek, H., y Korenblum, M. (1994). The relationship between life events during adolescence and affect and personality functioning. The Canadian Journal of Psychiatry, 39(6), 354-357.

VanIJzendoorn, M. H. (1995). Adult attachment representations, parental responsiveness, and infant attachment: A meta-analysis on the predictive validity of the Adult Attachment Interview. Psychological bulletin, 117(3), 387.

Warren, S. L., Huston, L., Egeland, B., y Sroufe, L. A. (1997). Child and adolescent anxiety disorders and early attachment. Journal of the American Academy of Child y Adolescent Psychiatry, 36(5), 637-644.

Weinfield, N. S., Ogawa, J. R., y Sroufe, L. A. (1997). Early attachment as a pathway to adolescent peer competence. Journal of Research on Adolescence, 7(3), 241-265.

Weinfield, N. S., Sroufe, L. A., Egeland, B., y Carlson, E. A. (1999). The nature of individual differences in infant-caregiver attachment. In J. Cassidy y P. R. Shaver (Eds.), Handbook of attachment: Theory, research, and clinical applications (pp. 68-88). New York: Guilford Press.

Westen, D., Nakash, O., Thomas, C., y Bradley, R. (2006). Clinical assessment of attachment patterns and personality disorder in adolescents and adults. Journal of consulting and clinical psychology, 74(6), 1065. 


\section{ANEXO I: Dimensiones del SENA en los grupos de edad propuestos por la prueba}

\begin{tabular}{|c|c|c|c|}
\hline DIMENSIONES & 3-6 años & $+6-12$ años & +12-18 años \\
\hline \multicolumn{4}{|l|}{ INDICES GENERALES } \\
\hline Índice global & $\mathrm{X}$ & $\mathrm{X}$ & $\mathrm{X}$ \\
\hline Índice de Problemas Emocionales & $\mathrm{X}$ & $\mathrm{X}$ & $\mathrm{X}$ \\
\hline Índice de problemas conductuales & $\mathrm{X}$ & $\mathrm{X}$ & $\mathrm{X}$ \\
\hline $\begin{array}{l}\text { Índice de problemas en funciones } \\
\text { ejecutivas }\end{array}$ & $\mathrm{X}$ & & $\mathrm{X}$ \\
\hline Índice de problemas contextuales & & $\mathrm{X}$ & $\mathrm{X}$ \\
\hline Índice de recursos personales & $\mathrm{X}$ & & $\mathrm{X}$ \\
\hline \multicolumn{4}{|l|}{ PROBLEMAS INTERIORIZADOS } \\
\hline Depresión & $\mathrm{X}$ & $\mathrm{X}$ & $\mathrm{X}$ \\
\hline Ansiedad & $\mathrm{X}$ & $\mathrm{X}$ & $\mathrm{X}$ \\
\hline Ansiedad social & $\mathrm{X}$ & & $\mathrm{X}$ \\
\hline Quejas somáticas & & & $\mathrm{X}$ \\
\hline Síntomas Postraumáticos & & & $\mathrm{X}$ \\
\hline Obsesión-compulsión & & & $\mathrm{X}$ \\
\hline \multicolumn{4}{|l|}{ PROBLEMAS EXTERIORIZADOS } \\
\hline Problemas de atención & $\mathrm{X}$ & $\mathrm{X}$ & $\mathrm{X}$ \\
\hline Hiperactividad-impulsividad & $\mathrm{X}$ & $\mathrm{X}$ & $\mathrm{X}$ \\
\hline Problemas del control de la ira & $\mathrm{X}$ & $\mathrm{X}$ & $\mathrm{X}$ \\
\hline Problemas de conducta & & $\mathrm{X}$ & \\
\hline Agresión & $\mathrm{X}$ & & $\mathrm{X}$ \\
\hline Conducta desafiante & $\mathrm{X}$ & & $\mathrm{X}$ \\
\hline Conducta antisocial & & & $\mathrm{X}$ \\
\hline \multicolumn{4}{|l|}{ OTROS PROBLEMAS } \\
\hline Consumo de sustancias & & & $\mathrm{X}$ \\
\hline Esquizotipia & & & $\mathrm{X}$ \\
\hline Problemas de conducta alimentaria & & & $\mathrm{X}$ \\
\hline Retraso en el desarrollo & $\mathrm{X}$ & & \\
\hline Comportamiento inusual & $\mathrm{X}$ & & \\
\hline
\end{tabular}




\begin{tabular}{|l|c|c|c|}
\hline \multicolumn{1}{|c|}{ DIMENSIONES } & $\mathbf{3 - 6}$ años & $\mathbf{+ 6 - 1 2}$ años & $\mathbf{+ 1 2 - 1 8}$ años \\
\hline PROBLEMAS CONTEXTUALES & & & \\
\hline Problemas familiares & & $\mathrm{X}$ & $\mathrm{X}$ \\
\hline $\begin{array}{l}\text { Problemas en la escuela } \\
\text { Problemas con compañeros }\end{array}$ & & $\mathrm{X}$ & $\mathrm{X}$ \\
\hline $\begin{array}{l}\text { ESCALAS DE VULNERABILIDADES } \\
\text { Regulación emocional }\end{array}$ & $\mathrm{X}$ & $\mathrm{X}$ & $\mathrm{X}$ \\
\hline $\begin{array}{l}\text { Autoestima } \\
\text { Rigidez }\end{array}$ & $\mathrm{X}$ & $\mathrm{X}$ \\
\hline $\begin{array}{l}\text { Aislamiento } \\
\text { Dificultad de apego }\end{array}$ & $\mathrm{X}$ & \\
\hline Integración y competencia social & $\mathrm{X}$ & & \\
\hline Conciencia del problema & & & $\mathrm{X}$ \\
\hline Inteligencia emocional & $\mathrm{X}$ & $\mathrm{X}$ \\
\hline Búsqueda de sensaciones & & & $\mathrm{X}$ \\
\hline
\end{tabular}

